\title{
Antimicrobial resistance and synergy in herbal medicine
}

\author{
Lorna Mundy, Barbara Pendry, M. Mukhlesur Rahman*
}

Medicines Research Group, School of Health, Sport and Bioscience, University of East London, Water Lane, Stratford, London E15 4LZ, UK. 


\begin{abstract}
Antimicrobial resistance (AMR) is a serious and growing threat to human health. The development of new antibiotics is limited and slow. The tradition of synergy in herbal medicine is being used as a source of research ideas. A literature review of antimicrobial research and plant synergy published in a five year period was carried out using online databases. The in vitro findings were that most of the research reported synergy both within plants and between plants and antibiotics. Whole plant extracts and combinations of compounds were shown to be more effective antimicrobials than isolated constituents. The discussion highlights that the in vitro herbal research findings are difficult to apply to practice and aren't progressing to clinical trials. Collaborative, innovative, inter-disciplinary clinical research is recommended.
\end{abstract}

Keywords: Antimicrobial resistance; herbal medicine; synergy 


\section{Abbreviations}

$\begin{array}{ll}\text { AMR } & \text { Antimicrobial Resistance } \\ \text { DOH } & \text { Department of Health } \\ \text { DEFRA } & \text { Department for Environment, Food and Rural Affairs } \\ \text { EBM } & \text { Evidence Based Medicine } \\ \text { ECDC } & \text { European Centre for Disease Prevention and Control } \\ \text { EHTPA } & \text { European Herbal and Traditional Practitioners Association } \\ \text { EPI } & \text { Efflux Pump Inhibitors } \\ \text { FIC } & \text { Fractional Inhibitory Concentration } \\ \text { MIC } & \text { Minimum Inhibitory Concentration } \\ \text { MDR } & \text { Multiple Drug Resistance } \\ \text { MRSA } & \text { Methicillin resistant Staphylococcus aureus } \\ \text { WHO } & \text { World Health Organisation }\end{array}$




\section{Introduction}

Health professionals, governments and international organisations are increasingly reporting the risks of antimicrobial resistance (AMR) to global health security. At a low estimate antibiotic resistance is currently causing 700,000 deaths worldwide annually, with this figure projected to reach 10 million by 2050 (O'Neil, 2014). The European Centre for Disease Prevention and Control (ECDPC) recently reported significant and increasing Multiple Drug Resistance (MDR) in Escherichia coli and Klebsiella pneumonia in more than a third of the countries that they report on (ECDPC, 2015). AMR increases the duration of illness and risk of death and has been predicted to make modern medical care impossible (Davies, 2013) with surgery and chemotherapy potentially becoming high risk interventions.

The World Health Organisation (WHO) reports that there are internationally high levels of AMR in common bacteria alongside limited understanding and uncoordinated surveillance of AMR (WHO, 2014). There have been just two new classes of antibiotics developed in the last 40 years. The development pipeline is slow and although two new Cephalosporin combinations are expected to be licensed in Europe soon for use in humans, AMR will also emerge for these (O'Neil, 2015). Bacterial mechanisms for resistance are innate but the high correlation between antibiotic use and AMR is clear (ECDPC, 2015). Further research, development of collaborative working, novel approaches to prevent and treat infections and the exploration of possibilities for enhancing immunity (in relation to infection by bacteria) including using prebiotics and probiotics have been recommended (DOH and DEFRA, 2013). Research and approaches for improving human immunity and resilience have been lacking (EUROCAM, 2014). WHO (2012) advises innovation and testing natural products to address AMR.

\subsection{Antimicrobial resistance}

Bacteria are prokaryotic micro-organisms, some of the earliest life forms, which created planetary conditions hospitable to animal life. There have been debates since the nineteenth century about whether diseases are caused by bacteria or the environment of a vulnerable, internally imbalanced body (EUROCAM, 2014). The dominant narrative of human relationship with bacteria has been the germ theory of disease which posited bacteria as enemies and motivated a war on them (Amyes, 2001). Kourtesi, et al. (2013) wrote of a subsequent mind-set that this war had later been won with the discovery of antibiotics. Antimicrobial refers to a substance with inhibitory action on either the growth or survival of micro-organisms (Davies, 2013). More specifically, antibiotics are naturally derived, largely antibacterial agents (Markovitch, 2010). A bacterium has 
intrinsic mechanisms for protection. The thick hydrophobic outer membrane of Gram negative bacteria and mycobacteria contributes to a greater resistance than Gram positive bacteria (Stavri et al., 2007). Efflux pumps remove toxins including clinical antibiotics out of the bacterium's cells. Increased production of efflux pumps is considered a main mechanism of bacterial resistance (Junio et al., 2011) particularly for multi-resistant Gram negative bacteria (Levy, 2002, Garvey et al., 2011and Betts et al., 2012.) Efflux pump inhibitors (EPI) are being researched to enable future efficacy of antibiotics but Buhner (2002) and Levy (2002) caution of the danger of this approach due to the ability of bacteria to quickly evolve into more harmful forms.

A variety of factors, including over reliance on antibiotics in healthcare and farming have caused bacteria to evolve and develop additional mechanisms of bacterial resistance in order to survive (Levy, 2002). It is well recorded but not fully understood how multiple drug resistance (MDR) can be developed in bacteria in a human or animal body through two weeks use of just a single antibiotic. 'It is almost as if bacteria strategically anticipate the confrontation of other drugs when they resist one' (Levy, 2002). The surprising extent of transferable drug resistance between different species of bacteria is understood to occur through horizontal genetic transfer of mobile traits (Smillie et al., 2011).

Blaser (2014), director of the Human Microbiome Project at New York University, describes how the trillions of microbes which have co-evolved to live with a species make up its microbiome. Blaser (2014) reports that $70-90 \%$ of cells in a human body are microbial symbionts, carrying out a range of important metabolic and protective functions. Gilbert et al. (2012) reported that in contemporary biology symbiosis is a core principle. They state that the old views of the immune system as 'defence,' 'weaponry' and 'self/non-self discrimination' are being inverted as it is increasingly understood that the microbiome co-creates the immune system (Gilbert, et al., 2012). In symbiotic biology, dynamic co-evolution with microbial symbionts is important to all mammals and research is finding ever greater microbial diversity and increasingly complex interrelating (Gilbert, et al., 2012). In all ecosystems diversity is crucial. After 30 years researching bacteria and human disease, Blaser (2014) argues that overused medical interventions (particularly antibiotics) have reduced the diversity of the human microbiome with damaging consequences to human health. In contrast to the understandings which led to the so called war on bacteria, contemporary research appears to be in the early stages of facilitating a paradigm shift in understandings of the human microbiome. 


\subsection{Plants and bacteria}

Plants can be described as complex, adaptive, synergistic systems (Niemeyer, et al., 2013). The low levels of infectious diseases found in wild plants, in contrast to crop plants (Hemaiswaya, et al., 2008) have been attributed in part to synergistic effects of multiple mildly antibacterial constituents and other hypothesised actions such as EPI (Buhner, 2012 \& Brown, 2015). Plants are understood to have co-evolved with pathogens and therefore developed effective chemical responses (Datta, et al., 2011). Plants in the wild are found to exhibit moderate antibacterial activity rather than entirely destroy the infectious species (Buhner, 2002). Plants and bacteria share a 'genetic fluidity' whereby they can respond to environmental stressors by rearranging their genotype (Buhner, 2002). Kourtesi, et al. (2013) state that plants respond to microbial threat significantly differently to the microbes which produce antibiotics, with plants instead evolving a complexity of synergists and toxins. Buhner (2012) observes the developing resistance of malaria parasites to artemisinin, a constituent of Artemisia spp. and argues that this will always happen with single constituent drugs whatever their origin.

\subsection{Synergy}

From a scientific perspective the challenge of synergy is that the concept, by its definition, lies outside the current belief that wholes, in this context a whole plant extract, can be understood by the isolation and analysis of its parts. Plant synergy is not considered a rational approach to the combination of molecules. Numerous mathematical models have been proposed in the quest for a quantitative measurement of synergy, the definition of which tends to be defined by the precise mathematical method used to demonstrate it. Berenbaum (1989) and Greco et al. (1995) review these methods which, because they were mainly designed to assess the interaction of pharmaceutical drug combinations, do not take into account the multiple compounds, actions, interactions and effects of whole herb preparations and formulae. Williamson (2001) in a review on plant synergy cited the isobole method as proposed by Berenbaum (1989) as the current method of choice.

Combination antimicrobial therapy, with some synergistic effects, is used successfully in chemotherapy, malaria and TB treatment and other specific scenarios but is not supported by the evidence for Gram negative bacterial infections (Tamma et al., 2015). It is recommended for Gram negative bacterial infections that the bacteria are rapidly identified and targeted in order to save future use of antibiotics (Tamma et al., 2015). 


\subsection{The synergistic approach}

Herbal medicine's uniqueness is due to its use of combinations of herbs and to the interactions (synergistic, additive or antagonistic) between constituents (Heinrich et al., 2012). Synergy is an effect of a combination of substances which is greater than would be expected by adding together their separate contributions (Williamson, 2001). There is currently much research aimed at identifying and isolating secondary metabolites of plants with antimicrobial activity (Rahman, 2014). Williamson (2001) discusses the limitations of isolated constituent research and advocates for more synergy research. The European Herbal and Traditional Practitioners Association (EHTPA) state that the synergism of phytoconstituents is significant in herbal pharmacokinetics and pharmacodynamics and may be a resource for responding to AMR (EHTPA, 2013). Heinrich, et al. (2012) state that although there is much interest in synergy and although it is held as a key factor in herbal medicine, it is not well written about or recorded. This paper aimed to review recent synergy research, in relation to AMR, both within herbal medicines and between plants and antibiotics.

\section{Methodology}

A literature search was conducted in May 2014 using keywords: antimicrobial OR antibiotic OR antibacterial AND synergy ${ }^{*}$ AND plant OR herb* OR natural. Electronic databases were searched: Ebsco HOST, Pub Med, Science Direct and the following journals: Planta Medica and Phytotherapy Research. Inclusion criteria were synergy research involving plants with antibacterial activity with or without antibiotics, published in peer reviewed journals in English between January 2010 and May 2014. It was outside the scope of this paper to cover the significant body of synergy research from Traditional Chinese Medicine or the research into the antimicrobial properties of essential oils. Reference lists of the identified research were hand searched and additional research traced online.

\section{Results}

\subsection{Literature review: antimicrobial research and plant synergy}

The following summary represents an overview of the literature that met the inclusion criteria for synergy research involving plants with antibacterial activity. Table 1 outlines methods for antimicrobial synergy research used or discussed in the research or literature. The studies which met the inclusion criteria are all in vitro. The disc diffusion assay and MIC measurement methods (Table 1) are used to explore in vitro assessments of antimicrobial activity of plant extract(s), 
fractions and pure compounds isolated from extracts or fractions in comparison with antibiotics. Synergy is tested using similar methods by applying a combination of antibiotic and plant extract or combination of compounds. Unlike pharmaceutical drugs where an exact dose of the active principle can be measured, the active principle in a whole plant extract is notoriously variable. To try and eliminate as many variables as possible, the use of plant extracts standardised to known active constituents is generally preferred for scientific validity (Williamson 2001).

\section{Table 1. Methods for researching antimicrobial synergy}

\begin{tabular}{|c|c|}
\hline Method & Process \\
\hline Disc diffusion & $\begin{array}{l}\text { Antibiotic and other solutions are tested on agar plates which have bacteria } \\
\text { evenly spread over them. Individual and combinations of constituents at } \\
\text { varying dilutions are applied to the agar on paper discs or in wells cut out of the } \\
\text { agar before incubation of plates. Zones of inhibition are measured (diameter in } \\
\text { mm) to show antimicrobial activity (Rahman, 2014). The size of the zones of } \\
\text { inhibition depends upon the diffusion of the active compounds into the agar. An } \\
\text { active non-polar compound may give only a small zone of inhibition because it } \\
\text { will not diffuse through the polar agar very well. Therefore the data is of limited } \\
\text { value for researching synergy as it cannot be used to compare the activities of } \\
\text { different compounds/extracts. Disc diffusion assay is comparatively straight } \\
\text { forward, time efficient, affordable and appropriate for researching plant } \\
\text { materials (Rahman, 2014). }\end{array}$ \\
\hline $\begin{array}{l}\text { Minimum inhibitory } \\
\text { concentration (MIC) } \\
\text { assay }\end{array}$ & $\begin{array}{l}\text { The minimum inhibitory concentration (MIC) is the lowest concentration of a } \\
\text { tested substance at which no bacterial growth can be seen (Bone and Mills, } \\
\text { 2013). For this purpose a method such as the disc diffusion assay should be } \\
\text { used, but even with this method, test substances need to be soluble in the } \\
\text { culture medium to display activity. }\end{array}$ \\
\hline $\begin{array}{l}\text { Fractional inhibitory } \\
\text { concentration (FIC) in } \\
\text { Checkerboards assay }\end{array}$ & $\begin{array}{l}\text { In the checker board assay (Orhan et al., 2005), an amount (e.g. } 50-100 \mu \mathrm{l}) \text { of } \\
\text { Mueller-Hinton broth is dispensed into all wells of the microdilution plates } \\
\text { followed by the addition of an antibiotic or first compound/ extract of the } \\
\text { combination which are serially diluted along the ordinate, whilst the compound/ } \\
\text { extract or the second sample serially diluted along the abscissa. Each well is } \\
\text { inoculated with a volume (e.g. } 100 \mu \mathrm{l} \text { ) of a bacteria followed by incubation under } \\
\text { standard conditions. The resulting checkerboard contains each combination } \\
\text { with wells containing the highest concentration of each sample at opposite } \\
\text { corners. Synergy is expressed as } \Sigma \text { FICs which is calculated as } \\
\Sigma \mathrm{FIC}=\text { FIC } 1+\mathrm{FIC} 2 \text {, where FIC } 1 \text { is the MIC of sample } 1 \text { in the combination/ } \\
\text { MIC of sample } 1 \text { alone, and FIC } 2 \text { is the MIC of sample } 2 \text { in the combination/ } \\
\text { MIC of sample } 2 \text { alone. The combination is synergistic if the } \Sigma F I C \text { is } \leq 0.5 \text {, } \\
\text { indifferent if the } \Sigma F I C \text { is }>0.5 \text { to }<2 \text {, and antagonistic if the } \Sigma F I C \text { is } \geq 2 \text {. }\end{array}$ \\
\hline The isobole method & $\begin{array}{l}\text { Both in vitro and in vivo bioassays can be used to demonstrate the isobole of a } \\
\text { mixture of two materials (two extracts, one extract plus a compound or two } \\
\text { compounds). This method provides a graph of } x \text { and y axis representing the dose } \\
\text { of the single individual components. (Fig. 1). The combined doses are expressed } \\
\text { by geometric points with coordinates which correspond to the individual doses } \\
\text { of each components in the mixture. An isobole is considered to be a line or curve } \\
\text { between points of the same activity. If the point representing a combination of }\end{array}$ \\
\hline
\end{tabular}


two substances forms a straight line with single points on the $x$-y axis, there is no interaction. If the point representing the effect of the combination lies below this line, the curve will be concave-up indicating that synergy is present. A point above this line produces a concave-down isobole indicating antagonism (Fig. 1). Wagner and Steinke (2004) have successfully employed this method to assess synergy between various mixtures of ginkgolide A and B (constituents of Ginkgo biloba) measured using the thrombocyte aggregation assay. Wagner (2004) comments however, that although this method may be suitable for doseresponse investigations with two-component containing mixtures, it is not a plausible method to be applied to herbal extract mixtures which would require detailed in vitro or in vivo comparative investigations with single constituents or mixtures and extract fractions or whole extracts to be performed.

Death kinetic (time-kill) assays

This method records antimicrobial activity over time and is clinically commended but not much used in research with plants. It is argued to be advantageous over MIC assays as it can show effects over time (van Vuuren and Viljoen, 2011). These assays would be used if a promising compound/extract is identified following MIC assays.

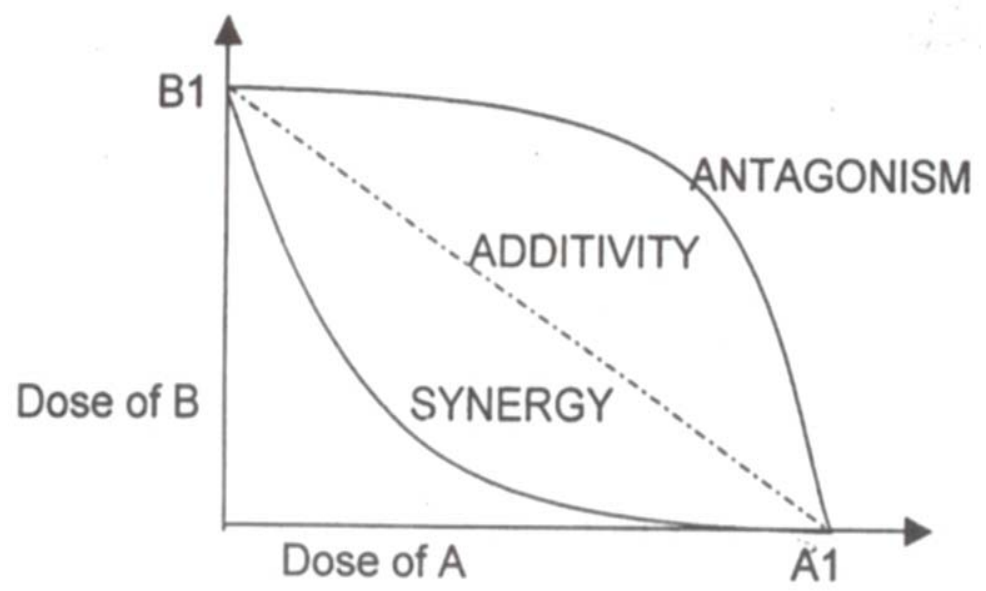

Figure 1. Isobologram illustrating additivity, synergy, and antagonism (Topps and Busia, 2005). $A 1$ and $B 1$ are the doses of the constituents $A$ and $B$ respectively, which produce an equal effect. The concave up isobole represents synergy. The concave down isobole represents antagonism. 


\subsection{Minimum inhibitory concentration (MIC)}

Lee and Lee (2010) used the disc diffusion assay to research individual antimicrobial effects and combined actions of Olea europea phenolics. No positive control antibiotics were included in the research methodology and the methodology was limited to disc diffusion studies so that the data are of limited value. The phenolics were tested individually and in combination in the ratios present in olive leaf extract. Zones of inhibition were measured against both Gram-negative bacteria such as Escherichia coli and Salmonella enteritidis and Gram-positive bacteria such as Bacillus cereus and Staphylococus aureus (Lee and Lee, 2010). A significant difference was detected in the antimicrobial activity whereby the phenolics mixture presented higher inhibition effects than the individual phenolics against Bacillus cereus and Salmonella enteritidis $(p<0.05)$. Further studies would need to be carried out to determine the potency of the compounds compared to known antimicrobials using a suitable quantitative assay and also to determine the degree of synergy between compounds. Ncube et al. (2012) researched three South African medicinal bulbs with both Gram-negative and Gram-positive bacteria. The antimicrobial interactions of extract combinations were assessed with microdilution assays and MIC measurements and then checkerboard method and FIC calculations (Ncube et al., 2012). The researchers found synergistic interactions indicated by the FIC indices for at least one of the extract combinations for each plant, including where one of the combinations had an FIC of 0.1 against Staphylococcus aureus although the individual MIC of each extract component against the same bacteria was $12.5 \mathrm{mg} / \mathrm{ml}$ (Ncube et al., 2012). The researchers reported more synergistic interactions with non-polar compounds than polar (Ncube et al., 2012) and linked this with previous findings of higher antimicrobial activity in non-polar extracts.

Adwan et al. (2010) used broth microdilution and synergy assay to find in vitro interactions between three Palestinian plant materials, five antibiotics and three strains of multi-drug resistant Gram negative bacteria Pseudomonas aeruginosa. The choice of bacteria was explained but not of plant species or plant parts. The growing conditions, harvesting and identification of plant materials were not transparent but the preparation of plant materials was explained. The authors used controls and measured the MIC of the antibiotics and plant extracts separately and in combination in duplicates. Adwan et al. (2010) found that the MIC decreased in the combinations particularly with Rhus coriara (seed) and attributed this to synergistic effects although the authors did not utilise the FIC index or the isobole method. For example Penicillin MIC >100, Rhus coriara MIC (3.125-1.563) x 103, Rhus coriara + Penicillin <0.012.2. The researchers went on to work on 
the identification of active constituents and highlighted the need for further work on the most effective plant-drug ratios, in vivo experiments and controlled clinical trials (Adwan et al., 2010).

Wang et al. (2013) conducted research with MRSA strains of Staphylococcus aureus and an ethanol extraction of a Tibetan medicinal plant Sophora moorcroftiana and found that constituents without antibacterial activity were significant synergists. Isolated compounds displayed less antibacterial activity than the extract (Wang et al., 2013), which could be attributed either to changes occurring during isolation procedures or synergy. By measuring the MIC values, calculating fractional inhibitory concentration (FIC) indices and conducting efflux assay tests it was concluded that the synergist compounds were genistein which had a moderate EPI action and diosmetin which had no EPI action with these bacteria but had a strong synergistic effect by a different mechanism (Wang et al., 2013).

\subsection{Fractional inhibitory concentration (FIC)}

Betts et al. (2012) tested tea polyphenols (theaflavin) with an antibiotic (Ampicillin) against clinical isolates of Gram negative multi drug resistant Stenotrophomonas maltophilia from hospital patients. Betts et al. (2012) found that when the antibiotic was used with theaflavin the MIC decreased from $12.5-22.9 \mu \mathrm{g} / \mathrm{mL}$ to $3.125-6.25 \mu \mathrm{g} / \mathrm{mL}$ to give a significant synergy FIC index of 0.22-0.35 (Table 1). The isobole method has been argued to be more accurate than the FIC index as it is more responsive to dose differentials (van Vuuren and Viljoen, 2011) but the research by Betts et al. (2012) did not use or mention this method. The authors did however highlight differences between the disc diffusion and microtitre assay, the former involving agar and therefore temperature and $\mathrm{pH}$ related variations in diffusion (Betts et al., 2012). In contrast, the microtitre assay (using a plate manufactured with wells) was preferred by the authors as it was not dependent on those variations and was rather more readily standardised and more accurate (Betts et al., 2012). Betts et al. (2012) concluded that further research is needed into mechanisms of action and emphasise the possibility for future effective clinical use of polyphenols with antibiotics in the context of antibiotic resistance.

Garvey et al. (2011) screened eighty four extracts of twenty one plants, 12 fractions thereof and 2 purified molecules for synergy with the antibiotic Ciprofloxacin against Salmonella enterica (Gram-negative bacteria). They used the microtitre method and presented FIC index values agreed to be indicative of synergy. Extracts of Melissa officinalis and Levisticum officinale were 
found to have independent antimicrobial activity against the Gram-negative bacteria and to have the greatest antibiotic potentiation effect (Garvey et al., 2011). They also investigated efflux pump inhibition capability of plant extracts. The highest efflux pump inhibition activity was revealed by the extracts of $L$. officinale. However, the synergistic activity of the plant extract was lost which might be either through alteration, inactivation or the separation of synergistic compounds as suggested by the authors.

\subsection{Synergy directed fractionation}

Bioassay guided fractionation is commonly carried out to find out the compound(s) responsible in the complex plant preparations. In this case, the active extracts are subjected to chromatographic techniques for the purification of compounds followed by their identification by spectroscopic methods. One drawback of this fractionation for studying botanical medicines is that it may not facilitate the identification of synergists which normally do not have the activity on their own but potentiate the activity of others. So during bioassay guided fractionation the potentiators may be overlooked. In order to address such challenges, Junio et al (2011) demonstrated synergy directed fractionation which involved the addition of an active compound at a fixed concentration to the extracts/ fractions which were subjected to synergy assays followed by the isolation of compounds from the extracts/ fractions of natural products. Junio et al (2011) used broth microdilulation antimicrobial checkerboard assays (Table 1) to evaluate the synergy of crude extracts in presence of berberine at a concentration of $5-300 \mu \mathrm{g} / \mathrm{ml}$. The crude extracts were fractionated and subjected to synergy testing in the presence of berberine using the same checkerboard assay. Among the fractions, a 16-fold decrease of the MICs (from 75 to 4.7) of berberine was observed in fraction 4 which was then further subjected to flash chromatography and HPLC whereby three flavonoids were isolated- sideroxylin, 8-desmethyl-sideroxylin and 6-desmethyl-sideroxylin. Junio, et al. (2011) reported that these_three flavonoids were synergistic in the antimicrobial activity of berberine against Staphylococcus aureus by inhibiting the Nor multidrug resistance pump. As Hydrastis canadensis roots are higher in alkaloids and the leaves higher in flavonoid synergists, the authors concluded that an extract against Staphylococcus aureus might be produced by mixing both roots and leaves and that the effectiveness of botanical medicine is the result of diverse constituents acting together Junio, et al. (2011). Although other authors considered possible and likely mechanisms of action Junio, et al. (2011) were the only ones to establish this through their methodology.

In this review, antibacterial synergy was reported for Olea europea (Lee and Lee, 2010), Hydrastis canadensis (Junio et al., 2012), Tulbaghia violacea, Hypoxis hemerocallidea and Merwilla 
plumbea (Ncube et al., 2012) and Sophora moorcroftiana Wang et al., 2013). Antibacterial synergy between plants and antibiotics was reported for: Rhus coriara (Adwan, et al., 2010), the polyphenol theaflavin (Betts, et al., 2012), Melissa officinalis and Levisticum officinale (Garvey et al., 2011). 


\section{Discussion}

Stavri et al. (2007) reported that most synergy research involving plants was on Gram-positive bacteria. Three of the studies in this review involved the clinically difficult to treat Gram-negative bacteria, two researched with Gram positive Staphylococcus aureus and two involved both Gramnegative and Gram-positive strains. Diffusion assay and measurement of zones of inhibition offer an indication of interactions but require cautious evaluation due to a multitude of influencing factors (van Vuuren and Viljoen, 2011), for example issues with solubility of compounds. As synergistic effects may occur at different concentration ratios, Heinrich et al. (2012) report that the isobole method is the agreed method of choice. Bone and Mills (2013) state that it is the only 'truly rigorous' evidence of synergy but that its complexity deters researchers. Junio et al. (2011) were the only researchers to plot an isobologram and their integration of several methodologies, (synergy directed fractionation), enabled the identification of active constituents whose synergistic activity might be missed by other methods (as they were not directly antimicrobial).

Bioassay guided isolation and fractionation (in vitro or in vivo) experiments (Table 1) identify the activities of a compound. However the results may be difficult to interpret due to compounds reacting with the extraction solvents though absorption, oxidation, degradation, evaporation where heat is used or the separation of unstable components. The activity of the extract may be progressively lost through the process but understanding why is hard to ascertain (Garvey, et al., 2011). Heinrich et al. (2012) poses the retention of biological activity following extraction as an important question as most extracts are complex and the concentration of active constituents may be low. Almost all the researchers were directly seeking or recommending in conclusions the identification of active compounds through bioactivity guided fractionation, however Junio, et al. (2011) argued that this approach can miss synergists with indirect actions such as potentiation (enhancing another constituent's action) and aim to engage with the complexity of herbal medicine through developing a new methodology.

The challenges of relating in vitro findings to in vivo and clinical practice are raised by many authors (Adwan, et al., 2010, Gertsch, 2011 and Mills, 2011). Absorption, metabolism and bioavailability are not predictable from in vitro findings. Niemeyer, et al. (2013) and others have suggested that the findings of reductionist approaches using processed plant parts and isolated constituents may not be generalisable to herbal medicine practice. All the research reviewed used dried plant extracts although there are researchers (Wright et al., 2010) and herbal medicine practitioners finding that traditional, fresh preparations have the best antimicrobial results (Buhner, 2013). 
Chemical complexity and the multi-targeted (polyvalent) nature of herbal medicine are understood as therapeutic strengths but make identification of active constituents a difficult goal (Bone and Mills, 2013). Most of the research reviewed here shows that whole plant extracts or combinations of compounds are more effective antimicrobials than isolated constituents (Junio et al., 2012), (Lee and Lee, 2010), (Ncube et al., 2012), Wang et al., 2013) and (Garvey et al., 2011). Combinations of non-specific mechanisms of action might create a more effective antimicrobial than an antibiotic (Kourtesi, et al. 2013). It has been argued that there is a risk of herbal medicine practice being reduced to practitioners prescribing based on the inconclusive findings of contemporary reductionist researchers (Niemeyer, et al., 2013). The presented findings highlight the challenges of reductionist methodologies in researching the complexity of plants. It is yet to be understood if plant sourced antimicrobials will be subject to the same AMR as the existing antibiotics. This paper agrees that there is a serious risk in attempting to meet AMR with the same paradigm of thinking which has created the situation.

\section{Conclusion}

This paper reports international findings of antimicrobial synergy within plants and between plants and antibiotics. Laboratory methodologies for plant based antimicrobial synergy research are shown to be developing but in vitro herbal research isn't progressing into clinical studies. The discussed methodologies and findings highlight the different paradigms and values in healthcare and the political context within which research and healthcare exist.

Contemporary understandings of complex systems science, symbiosis and the microbiome are pointing to new ways of seeing and responding to AMR. It is recommended that these perspectives and their developing research methodologies are supported by scientists, policy makers and herbal medicine practitioners. Synergy in herbal medicine can be seen to be significant in the context of AMR not just because of antimicrobials and synergists but because it adds to these systemic ways of knowing. From these emerging understandings it is clear that both the use and development of antimicrobials need careful consideration for whole systems health. The findings presented in this paper suggest that healthcare could learn much from plants and herbal medicine traditions about co-evolving, diversity, adaptability and the complexities of synergy. Collaborative, innovative, inter-disciplinary clinical research is recommended to meet the challenge of AMR. 


\section{References}

Brown A. Studies on bacterial efflux pump inhibitors in land plants. Doctoral dissertation, The University of North Carolina at Greensboro; 2015.

Adwan G, Abu-Shanab B, Adwan K. Antibacterial activities of some plant extracts alone and in combination with different antimicrobials against multidrug-resistant Pseudomonas aeruginosa strains. Asian Pac J Tropical Med 2010; 3: 266-269.

Amyes SGB. Magic Bullets, Lost Horizons: The Rise and Fall of Antibiotics. London: Taylor and Francis; 2001.

Berenbaum MC. What is synergy? Pharmacolog Rev 1989;41: 93-141.

Betts JW, Murphy C, Kelly SM, Haswell SJ, Synergistic antibacterial effects of theaflavin in combination with ampicillin against hospital isolates of Stenotrophomonas maltophilia. $J$ Microbiol Biotechnol Food Sci 2012;2:1068-78.

Blaser MJ. Missing Microbes: How Killing Bacteria Creates Modern Plagues. London: Oneworld; 2014.

Bone K, Mills S. Principles and Practice of Phytotherapy: Modern Herbal Medicine. $2^{\text {nd }}$ ed. London: Churchill Livingston Elsevier; 2013.

Buhner SH. Herbal Antibiotics: Natural Alternatives for Treating Drug-Resistant Bacteria. $2^{\text {nd }}$ ed. North Adams: Storey Publishing; 2012.

Buhner SH. The Lost Language of Plants: The Ecological Importance of Plant Medicines to Life on Earth. Vermont: Chelsea Green Publishing; 2002.

Datta S, Pal NK, Nandy AK. Inhibition of the emergence of multi drug resistant Staphylococcus aureus by Withania somnifera root extracts. Asian Pac J Tropical Med 2011: 4: 917-920.

Davey P. What is antimicrobial stewardship? The current landscape. The British Society for Antimicrobial Chemotherapy Spring Meeting on Antimicrobial stewardship in human and animal health. Royal College of Physicians, London; 2014.

Davies SC. 2013. The Drugs Don't Work: A Global Threat. London: Penguin.

DEFRA, 2014. Antimicrobial Resistance (AMR): information and resources.[Online] Available at: $<$ https://www.gov.uk/government/collections/antimicrobial-resistance-amr-information-andresources> [Accessed July 2014]

DOH and DEFRA, 2013. UK 5 Year Antimicrobial Resistance Strategy 2013 to 2018. [Online] Available at:

$<$ https://www.gov.uk/government/uploads/system/uploads/attachment_data/file/244058/2013090 2_UK_5_year_AMR_strategy.pdf> [Accessed June 2014]

EHTPA, 2013. A Paper from the European Herbal and Traditional Medicine Practitioners Association for the House of Commons Select Committee on Science and Technology's antimicrobial resistance (AMR) inquiry. Tewkesbury: EHTPA. 
ECDPC. 2015. Annual epidemiological report 2014 - Antimicrobial resistance and healthcareassociated infections. Available at:

http://ecdc.europa.eu/en/publications/ layouts/forms/Publication DispForm.aspx?List=4f55ad51 -4aed-4d32-b960-af70113dbb90\&ID=1292\#sthash.m2RGdAIQ.dpuf

EUROCAM, 2014. The role of Complementary and Alternative Medicine (CAM) in reducing the problem of antimicrobial resistance. Available at: http://www.cam-

europe.eu/dms/files/Position Papers/EUROCAM PositionPaper CAM and AMR November20 $\underline{14 . p d f}$

Falcão--Silva VS, Silva DA, Souza MDFV, Siqueira-Junior JP. Modulation of drug resistance in Staphylococcus aureus by a kaempferol glycoside from Herissantia tiubae (Malvaceae).

Phytother Res 2009; 23: 1367-1370.

Garvey MI, Rahman MM, Gibbons S, Piddock LJ, Medicinal plant extracts with efflux inhibitory activity against Gram-negative bacteria. Int J Antimicrobial Agents 2011; 37: 145-151.

Gertsch J. Botanical drugs, synergy, and network pharmacology: forth and back to intelligent mixtures. Planta Med 2011;77: 1086.

Gilbert SF. Symbiosis as the way of eukaryotic life: The dependent co-origination of the body. J Biosciences 2014; 39: 201-209.

Gilbert SF, Sapp J, Tauber Al. A symbiotic view of life: we have never been individuals. The Quarterly Rev Biol 2012; 87: 325-341.

Greco WR, Bravo G, Parsons JC. The search for synergy: a critical review from a response surface perspective. Pharmacol Rev 1995; 47:331-385.

Heinrich M, Barns J, Gibbons S, Williamson EM. Fundamentals of Pharmacognosy and Phytotherapy. $2^{\text {nd }}$ ed. London: Churchill Livingstone Elsevier; 2012.

Hemaiswarya S, Kruthiventi AK, Doble M. Synergism between natural products and antibiotics against infectious diseases. Phytomed 2008.;15: 639-652.

Junio HA, Sy-Cordero AA, Ettefagh KA, Burns JT, Micko KT, Graf TN, Cech N B. Synergydirected fractionation of botanical medicines: a case study with goldenseal (Hydrastis canadensis). J Nat Prod 2011; 74: 1621-1629.

Kourtesi C, Ball AR, Huang YY, Jachak SM, Vera DMA, Khondkar P, Gibbons S, Hamblin MR,Tegos G. P.. Microbial Efflux Systems and Inhibitors: Approaches to Drug Discovery and the Challenge of Clinical Implementation. The Open Microbiol J, 2013; 7: 34.

Levy SB. The Antibiotic Paradox: How the Misuse of Antibiotics Destroys their Curative Powers. $2^{\text {nd }}$ ed. Cambridge (USA): Perseus Publishing; 2002.

Markovitch H. (ed.) 2010. Black's Medical Dictionary. 42 ${ }^{\text {nd }}$ Ed. London: A \& C Black Publishers; 2010. 
Mills SY. 2011. Research in herbal medicine. In: Lewith GT, Jonas BW, Walach H. (eds.) Clinical Research in Complementary Therapies: Principles, Problems and Solutions. $2^{\text {nd }}$ ed. London: Churchill Livingston Elsevier; 2011.

Ncube B, Finnie JF, Van Staden J. In vitro antimicrobial synergism within plant extract combinations from three South African medicinal bulbs. J Ethnopharmacol 2012; 139: 81-89.

Niemeyer K, Bell IR, Koithan M, Traditional knowledge of Western herbal medicine and complex systems science. J. Herbal Med 2013; 3: 112-119.

O'Neil. The Review on Antimicrobial Resistance. Antimicrobial Resistance: Tackling a Crisis for the Health and Wealth of Nations. 2014.

O'Neil. The Review on Antimicrobial Resistance. Securing new Drugs for Future Generations: The Pipeline of Antibiotics. 2015.

Orhan, G., Bayram, A., Zer, Y. and Balci, I. (2005) Synergy Tests by E Test and Checkerboard Methods of Antimicrobial Combinations against Brucella melitensis. J Clin Microbiol. 43: 140143.

Rahman MM. Antimicrobial secondary metabolites- extraction, isolation, identification and bio assay. In: Mukherjee, P. (ed), Evidence-Based Validation of Herbal Medicine Amsterdam, Oxford and, Waltham: Elsevier, pp 495-513.; 2014.

Shami AM M, Philip K, Muniandy S. Synergy of antibacterial and antioxidant activities from crude extracts and peptides of selected plant mixture. BMC Complementary and Alternative Med, 2013; 13:360.

Smillie C S, Smith MB, Friedman J, Cordero OX, David L A, Alm EJ. Ecology drives a global network of gene exchange connecting the human microbiome. Nature 2011; 480, 241-244.

Stavri M, Piddock LJ., Gibbons S. Bacterial efflux pump inhibitors from natural sources. J Antimicrob Chemother 2007; 59:1247-1260.

Tamma PD, Cosgrove SE, Maragakis LL. Combination therapy for treatment of infections with gram-negative bacteria. Clinical microbiology reviews. 2012 Jul 1;25(3):450-70.

Topps, G and Busia, K. The concept of synergy: time for a new paradigm? [online]. Australian Journal of Medical Herbalism, Vol. 17, No. 2, 2005: 45-54.

van Vuuren S, Viljoen A. Plant-based antimicrobial studies-methods and approaches to study the interaction between natural products. Planta Med 2011; 77: 1168.

Wagner, H. \& Steinke, B. (2004). 'Synergy effects of a mixture of ginkalide A+B in a thrombocyte aggregation inhibiting assay'. Phytomedicine 11 (2-3)

Wang SY, Sun ZL, Liu T, Gibbons S, Zhang WJ, Qing M. Flavonoids from Sophora moorcroftiana and their Synergistic Antibacterial Effects on MRSA. Phytother Res. 2014.

WHO, 2012. Evolving Threat of Antimicrobial Resistance: Options for Action. [Online] Available at: <http://www.who.int/patientsafety/implementation/amr/publication/en/> [Accessed March 2014]. 
WHO, 2014. Antimicrobial Resistance: Global Report on Surveillance. [Online] Available at: <http://www.who.int/drugresistance/documents/surveillancereport/en/> [Accessed July 2014].

WHO, 2014. Antimicrobial Resistance Factsheet. [Online] Available at:

<http://www.who.int/mediacentre/factsheets/fs194/en/> [Accessed June 2014].

Williamson EM. Synergy and other interactions in phytomedicines. Phytomed 2001; 8: 401-409.

Wright, C.W., Linley, P.A., Brun, R., Wittlin, S. and Hsu, E. Ancient Chinese Methods are remarkably effective for the preparation of artemisinin-rich extracts of Qing Hao with potent antimalalarial activity. Molecules, 15:804-812, 2010.

Conflict of interest

The authors declare that there is no conflict of interests regarding the publication of this article. 